Pacific Journal of Mathematic 


\section{INVARIANCE FOR LINEAR SYSTEMS OF ORDINARY DIFFERENTIAL EQUATIONS}

\section{Al KeLLeY}

In studying the existence and smoothness of invariant manifolds arising from nonlinear, perturbed systems of ordinary differential equations, one encounters the study of certain linear (in $x$ ), perturbation problems of the type

$$
\begin{aligned}
& \dot{\theta}=a+\varepsilon b(\theta, \varepsilon) \\
& \dot{x}=(A+\varepsilon B(\theta, \varepsilon)) x
\end{aligned}
$$

where $\theta$ and $x$ are vectors, $A$ and $B$ are matrices, $b$ and $B$ are multiply periodic in $\theta$, and $\varepsilon$ is a perturbation parameter. Assuming $A$ is a constant matrix consisting of square submatrices on the diagonal,

$$
A=\operatorname{diag}\left(A_{11}, \cdots, A_{n n}\right),
$$

with the maximum of the real parts of the eigenvalues of $A_{j j}$ less than the minimum of the real parts of the eigenvalues of $A_{k k}$ for $1 \leqq j<k \leqq n$; we construct a change of variables which reduces $B$ to similar diagonal form.

For perturbed systems of nonlinear ordinary differential equations in a neighborhood of an invariant manifold, the existence and smoothness of the center-stable, center, and center-unstable manifolds is proved in $\S 6$ of [3]. The method of proof used will also show the existence of other invariant manifolds, but for nonlinear systems the situation is not as simple as the associated linear problem with regard to finding invariant manifolds. $R$. Venti [7] has given linearization results for nonlinear systems of differential equations near a critical point. The results of this paper can be regarded as a first step in obtaining similar linearization results for nonlinear systems near an invariant manifold.

The techniques of this paper are based on those used by Y. Sibuya [5], [6]. Sibuya treats time-varying perturbation problems where the perturbation parameter enters in an analytic way. In $\S 3$ of this paper we consider $C^{k}(1 \leqq k<\infty)$, $\theta$-varying perturbation problems with $\theta$ representing the many-dimensional coordinates of some invariant manifold. In $\S 4$ we give a counter-example to an analytic change of variables procedure, and then modify the procedure appropriately.

For linear systems of ordinary differential equations of the type

$$
\dot{x}=(A+\varepsilon B(t, \varepsilon)) x
$$

(see (1) below with $\operatorname{dim} \theta=1, \dot{\theta}=1$, for details), where the matrix $B$ 
is periodic in $t$, the theory presented in $\S 3$ and $\S 4$ below applies. However, for this periodic, time-dependent, perturbation problem, much more can be said. See, for example, Lemmas 4, 5, 6 in Sibuya [6] or Chapter 8 in Hale [2].

2. Notation. If $G=G(g)$ is a smooth, vector valued function of the vector $g$, then $G_{g}$ represents the usual Jacobian matrix of partial derivatives. If $H=H(g)$ is a smooth, matrix valued function of the vector $g=\left(g_{1}, \cdots, g_{n}\right)$, then $H_{g}$ represents an array of all possible first order derivatives,

$$
H_{g}=\left(\frac{\partial}{\partial g_{1}} H, \frac{\partial}{\partial g_{2}} H, \cdots, \frac{\partial}{\partial g_{n}} H\right) \text {. }
$$

The norm $|\cdot|$ represents the euclidean norm on vectors and the operator norm on matrices; $\langle\cdot, \cdot\rangle$ represents the usual inner product on pairs of vectors. If $\rho$ is an $n$-tuple of nonnegative integers, then $D_{g}^{\rho}=\partial^{|\rho|} / \partial^{\rho_{1}} g_{1} \cdots \partial^{\rho_{n}} g_{n}$ where $|\rho|=\rho_{1}+\cdots+\rho_{n}$. (The "norm" on $\rho$ is not euclidean which we justify by not considering $\rho$ to be a vector.)

3. Invariance for linear systems. Consider the real, $C^{1}$ system of ordinary differential equations

$$
\begin{aligned}
& \dot{\theta}=a+\varepsilon b(\theta, \varepsilon) \\
& \dot{x}=(A+\varepsilon B(\theta, \varepsilon)) x
\end{aligned}
$$

where $\theta, x, a, b$ are real vectors; $a$ is a constant vector; $\varepsilon$ is a real perturbation parameter; $b$ is defined and $C^{1}$ on

$$
N_{\delta}=\{(\theta, \varepsilon) \mid \theta \text { arbitrary, }|\varepsilon|<\delta\} ;
$$

$b$ and $b_{(\theta, \varepsilon)}$ are uniformly bounded on $N_{\delta} ; A$ is a real, constant, square matrix partitioned as follows:

$$
\begin{aligned}
& A=\left(A_{j k}\right) \text { with } A_{j k}=0 \text { for } j \neq k, \text { equivalently } \\
& A=\operatorname{diag}\left(A_{11}, A_{22}, \cdots, A_{n n}\right)
\end{aligned}
$$

the sub-matrices $A_{j j}(j=1, \cdots, n)$ on the diagonal are square but not necessarily of the same dimension; the sub-matrices on the diagonal have the property that the maximum of the real parts of the eigenvalues of $A_{j j}$ is less than the minimum of the real parts of the eigenvalues of $A_{k k}$ when $j<k$ : symbolically

$$
A_{11}<A_{22}<\cdots<A_{n n}
$$

where the order relation $<$ reflects the ordering of the real parts of the eigenvalues of the sub-matrices; $B$ is a real, $C^{1}$, square matrix 
defined on $N_{\delta} ; B$ and $B_{(\theta, \varepsilon)}$ are uniformly bounded on $N_{\delta}$.

THEOREM 1. For system (1) there exists a unique $C^{1}$ change of variables

$$
x=(I+\varepsilon P(\theta, \varepsilon)) y
$$

such that

$$
\dot{y}=(A+\varepsilon Q(\theta, \varepsilon)) y
$$

where $I$ is the identity matrix; $P$ and $Q$ are real, $C^{1}$ matrices defined on $N_{\delta_{1}}$ for $0<\delta_{1} \leqq \delta$ sufficiently small; $P, P_{(\theta, \varepsilon)}, Q, Q_{(\theta, \varepsilon)}$ are uniformly bounded on $N_{\delta_{1}}$; with $P$ and $Q$ partitioned into sub-matrices similar to $A$,

$$
\begin{array}{ll}
P_{j j}(\theta, \varepsilon) \equiv 0 & (j=1, \cdots, n) \\
Q_{j_{k}}(\theta, \varepsilon) \equiv 0 & (j, k=1, \cdots, n ; j \neq k) ;
\end{array}
$$

if system (1) has multiple period $\omega$ in $\theta$, then the change of variables (3) and the transformed system (4) also have multiple period $\omega$ in $\theta$.

Proof. Assuming the change of variables exists, we differentiate both sides of (3),

$$
\dot{x}=\varepsilon \dot{P} y+(I+\varepsilon P) \dot{y},
$$

and from (1), (3), (4) we have

$$
(A+\varepsilon B)(I+\varepsilon P) y=\varepsilon \dot{P} y+(I+\varepsilon P)(A+\varepsilon Q) y,
$$

which leads to the matrix equation

$$
\dot{P}=A P-P A+B-Q+\varepsilon B P-\varepsilon P Q \text {. }
$$

Let $\psi=\psi(t, \theta, \varepsilon)$ be the unique solution of the $\theta$-equation in (1) with initial condition $\theta$ at $t=0$. This solution exists and is $C^{1}$ on

$$
\tilde{N}_{\delta}=\left\{(t, \theta, \varepsilon) \mid-\infty<t<+\infty,(\theta, \varepsilon) \in N_{\delta}\right\} .
$$

Rather than expressing $\dot{P}$ on the left side of (6) as $P_{\theta}\{a+\varepsilon b(\theta, \varepsilon)\}$, we consider

$$
\dot{P}=\frac{d}{d t} P(\psi(t, \theta, \varepsilon), \varepsilon) ;
$$

in words, we interpret $\dot{P}$ as the "derivative along the solution curve". (See the proof of Theorem 1 in [4] for a similar example of this notion.) From (5) and (6) 
(7a)

$$
\begin{aligned}
Q_{j j}= & B_{j j}+\varepsilon \sum_{l=1}^{n} B_{j l} P_{l j} \quad(j=1, \cdots, n) \\
\dot{P}_{j k}= & A_{j j} P_{j k}-P_{j k} A_{k k}+B_{j k} \\
& +\varepsilon \sum_{l=1}^{n} B_{j l} P_{l k}-\varepsilon P_{j k} Q_{k k} \quad(j, k=1, \cdots, n ; j \neq k) .
\end{aligned}
$$

Conversely, we observe that a $C^{1}$ solution of (7) which is uniformly bounded on some $N_{\delta_{1}}$ will yield the change of variables (3) and the transformed system (4). To solve (7) by iteration define $P^{0} \equiv 0$ and define $Q^{\nu}, H^{\nu}, P^{\nu}$ iteratively as follows. (Super-scripts designate steps of the iteration, not powers of the matrices.)

$$
\begin{aligned}
Q_{j j}^{\nu} & =B_{j j}+\varepsilon \sum_{l=1}^{n} B_{j l} P_{l j}^{\nu} \\
Q_{j k}^{\nu} & \equiv 0
\end{aligned}
$$

$$
(1 \leqq j, k \leqq n ; j \neq k)(\nu=0,1,2, \cdots)
$$

$$
\begin{aligned}
& H^{\nu}=B P^{\nu-1} P^{\nu-1} Q^{\nu-1}, \text { so that in particular } \\
& H_{j k}^{\nu}=\sum_{l=1}^{n} B_{j l} P_{l k}^{\nu-1}-P_{j k}^{\nu-1} Q_{k k}^{\nu-1}
\end{aligned}
$$

$$
(1 \leqq j, k \leqq n ; j \neq k)(\nu=1,2, \cdots)
$$

$$
\begin{aligned}
& P_{j j}^{\nu} \equiv 0 \\
& \dot{P}_{j k}^{\nu}=A_{j j} P_{j k}^{\nu}-P_{j k}^{\nu} A_{k k}+B_{j k}+\varepsilon H_{j k}^{\nu} \\
& \quad(1 \leqq j, k \leqq n ; j \neq k)(\nu=1,2, \cdots) .
\end{aligned}
$$

The unique bounded solution $P_{j k}^{\nu}$ of the differential equation in (8c) is given by

$$
P_{j k}^{\nu}(\theta, \varepsilon)=\int_{ \pm \infty}^{0} e^{-A_{j j} \sigma}\left\{B_{j k}(\psi(\sigma, \theta, \varepsilon), \varepsilon)+\varepsilon H_{j k}^{\nu}(\psi(\sigma, \theta, \varepsilon), \varepsilon)\right\} e^{A_{k k} \sigma} d \sigma
$$

where the lower limit of integration is chosen $+\infty$ for $A_{k k}<A_{j j}$ and $-\infty$ for $A_{j j}<A_{k k}$ with $<$ the order relation in (2). If we assume that $H^{\nu}$ is a known $C^{1}$ function of $(\theta, \varepsilon)$ on some $N_{\delta_{1}}$ with $H^{\nu}, H_{(\theta, \varepsilon)}^{\nu}$ uniformly bounded on $N_{\delta_{1}}$, and if we assume that the infinite integral in (9) converges, then by replacing $\theta$ in (9) by $\psi(t, \theta, \varepsilon)$ we easily check that $P_{j k}^{\nu}(\psi(t, \theta, \varepsilon), \varepsilon)$ satisfies (8c). Beginning with $P^{0} \equiv 0$ we want to show that (8) and (9) determine $Q^{\nu}, H^{\nu}, P^{\nu}(\nu=1,2, \cdots)$ iteratively as $C^{1}$ functions of $(\theta, \varepsilon)$ defined on some $N_{\delta_{1}}$ with $Q^{\nu}, H^{\nu}, P^{\nu},\left(Q^{\nu}, H^{\nu}, P^{\nu}\right)_{(\theta, \varepsilon)}$ uniformly bounded on $N_{\delta_{1}}$. We will need estimates on the rates of growth for $e^{A_{j} j^{t}}(j=1, \cdots, n), \psi_{\theta}(t, \theta, \varepsilon), \psi_{\varepsilon}(t, \theta, \varepsilon)$.

Associated with each $A_{j j}$ is its real canonical form $\hat{A}_{j j}$,

$$
\hat{A}_{j j}=J_{j j}^{-1} A_{j j} J_{j j} \quad(j=1, \cdots, n),
$$

with the "off-diagonalizable" terms of $\hat{A}_{j j}$ arbitrarily small. The ordering (2) means there exists real numbers 


$$
\mu_{1} \leqq \tilde{\mu}_{1}<\mu_{2} \leqq \tilde{\mu}_{2}<\cdots<\mu_{n} \leqq \tilde{\mu}_{n}
$$

such that

$$
\mu_{j}|p|^{2} \leqq\left\langle\hat{A}_{j j} p, p\right\rangle \leqq \tilde{\mu}_{j}|p|^{2} \quad(j=1, \cdots, n)
$$

holds for all vectors $p$ (restricted for each $j$ to the appropriate dimension $\left.\left(\operatorname{dim} \hat{A}_{j j}=\operatorname{dim} p \times \operatorname{dim} p\right)\right)$. See [1] page 341 for details concerning the real canonical form of a matrix. From (12a) we conclude for $(j=1, \cdots, n)$

$$
\begin{array}{ll}
e^{\mu_{j} t} \leqq\left|e^{\hat{A}_{j} t}\right| \leqq e^{\tilde{\mu}_{j} t} & (0 \leqq t<+\infty) \\
e^{\mu_{j} t} \geqq\left|e^{\hat{A}_{j} t}\right| \geqq e^{\tilde{\mu}_{j} t} & (-\infty<t \leqq 0) .
\end{array}
$$

From (1) for $(t, \theta, \varepsilon) \in \tilde{N}_{\delta}$

$$
\begin{aligned}
\dot{\psi}_{\theta}(t, \theta, \varepsilon)= & \varepsilon b_{\theta}(\psi(t, \theta, \varepsilon), \varepsilon) \psi_{\theta}(t, \theta, \varepsilon) \\
\dot{\psi}_{\varepsilon}(t, \theta, \varepsilon)= & b(\psi(t, \theta, \varepsilon), \varepsilon)+\varepsilon b_{\theta}(\psi(t, \theta, \varepsilon), \varepsilon) \psi_{\varepsilon}(t, \theta, \varepsilon) \\
& +\varepsilon b_{\varepsilon}(\psi(t, \theta, \varepsilon), \varepsilon) \\
\psi_{\theta}(0, \theta, \varepsilon) \equiv & I \text { (identity), } \psi_{\varepsilon}(0, \theta, \varepsilon) \equiv 0
\end{aligned}
$$

which yields

$$
\begin{aligned}
& \left|\dot{\psi}_{\theta}\right| \leqq|\varepsilon| K_{1}\left|\psi_{\theta}\right| \\
& \left|\dot{\psi}_{\varepsilon}\right| \leqq K_{1}+|\varepsilon| K_{1}\left|\psi_{\varepsilon}\right| \\
& \left|\psi_{\theta}(0, \theta, \varepsilon)\right| \equiv 1, \quad\left|\psi_{\varepsilon}(0, \theta, \varepsilon)\right| \equiv 0,
\end{aligned}
$$

where $K_{1}$ is a sufficiently large positive constant; more specifically we may take

$$
K_{1}=(1+\delta) \max \left\{\sup _{(\theta, \varepsilon) \in N_{\delta}}|b(\theta, \varepsilon)|, \sup _{(\theta, \varepsilon) \in N_{\delta}}\left|b_{(\theta, \varepsilon)}(\theta, \varepsilon)\right|\right\} .
$$

From (13) and the Hale inequality (see Lemma 2 in [3])

$$
\begin{aligned}
& \left|\psi_{\theta}(t, \theta, \varepsilon)\right| \leqq e^{|\varepsilon| K_{1}|t|} \\
& \left|\psi_{\varepsilon}(t, \theta, \varepsilon)\right| \leqq K_{1}|t| e^{|\varepsilon| K_{1}|t|}
\end{aligned}
$$

holds for all $(t, \theta, \varepsilon) \in \tilde{N}_{\dot{\delta}}$.

In what follows $K_{2}, K_{3}, \cdots$ (a finite number of $K$ 's) will designate sufficiently large positive constants. If $j<k$, we have from (9), (10), (12)

$$
\begin{aligned}
\left|P_{j k}^{\nu}(\theta, \varepsilon)\right| \leqq & \int_{-\infty}^{0}\left|J_{j j}\right|\left|e^{-\hat{A}_{j j} \sigma}\right|\left|J_{j j}^{-1}\right| \\
& \cdot\left\{\left|B_{j k}(\psi(\sigma, \theta, \varepsilon), \varepsilon)\right|+|\varepsilon| \mid H_{j k}^{\nu}(\psi(\sigma, \theta, \varepsilon), \varepsilon)[\}\right. \\
& \cdot\left|J_{k k}\right|\left|\hat{e}^{A} k_{k k} \sigma\right|\left|J_{k k}^{-1}\right| d \sigma \\
\leqq & \int_{-\infty}^{0} K_{2} e^{-\tilde{\mu}_{j} \sigma}\{\cdots\} K_{2} e^{\mu_{\grave{k}} \sigma} d \sigma
\end{aligned}
$$


where $K_{2}$ is chosen sufficiently large so that

$$
\left|J_{j j}\right|\left|J_{j j}^{-1}\right| \leqq K_{2} \quad(j=1, \cdots, n) .
$$

A similar inequality holds for the case $j>k$. From (11), (15) we conclude that (8), (9) determine $Q^{\nu}, H^{\nu}, P^{\nu}$ iteratively as continuous functions of $(\theta, \varepsilon)$ defined and uniformly bounded on $N_{\delta}$.

Suppose we have shown $P^{\nu-1}$ to be $C^{1}$ on some $N_{\delta_{1}}\left(\delta_{1}\right.$ yet to be determined) with $P^{\nu-1}, P_{(\theta, \varepsilon)}^{\nu-1}$ uniformly bounded on $N_{\delta_{1}}$. Then $Q^{\nu-1}$, $H^{\nu}$ given by $(8 \mathrm{a}, \mathrm{b})$ will also be $C^{1}$ on $N_{\delta_{1}}$ with $Q^{\nu-1}, H^{\nu},\left(Q^{\nu-1}, H^{\nu}\right)_{(\theta, \varepsilon)}$ uniformly bounded on $N_{\delta_{1}}$. Let $\theta=\left(\theta_{1}, \cdots, \theta_{m}\right)$ so that $\theta_{l}$ represents the $l^{\text {th }}$-component of $\theta$ and $m=\operatorname{dim} \theta$. If $\partial / \partial \theta_{l}$ commutes with integration, then differentiating the right side of (9) we obtain

$$
\begin{aligned}
\frac{\partial}{\partial \theta_{l}} \int_{ \pm \infty}^{0} e^{-A_{j j} \sigma}\left[B_{j k}(\psi, \varepsilon)+\varepsilon H_{j k}^{\nu}(\psi, \varepsilon)\right] e^{A_{k k} \sigma} d \sigma \\
=\int_{ \pm \infty}^{0} e^{-A_{j j} \sigma}\left[\left\{\left(\frac{\partial}{\partial \theta_{1}} B_{j k}\right)(\psi, \varepsilon)\right\} \frac{\partial}{\partial \theta_{l}} \psi_{1}+\cdots\right. \\
\quad+\left\{\left(\frac{\partial}{\partial \theta_{m}} B_{j k}\right)(\psi, \varepsilon)\right\} \frac{\partial}{\partial \theta_{l}} \psi_{m}+\varepsilon\left\{\left(\frac{\partial}{\partial \theta_{1}} H_{j k}^{\nu}\right)(\psi, \varepsilon)\right\} \frac{\partial}{\partial \theta_{l}} \psi_{1} \\
\left.+\cdots+\varepsilon\left\{\left(\frac{\partial}{\partial \theta_{m}} H_{j k}^{\nu}\right)(\psi, \varepsilon)\right\} \frac{\partial}{\partial \theta_{l}} \psi_{m}\right] e^{A_{k k} \sigma} d \sigma .
\end{aligned}
$$

If $\partial / \partial \varepsilon$ commutes the integration, then differentiating the right side of (9) we obtain

$$
\begin{aligned}
& \frac{\partial}{\partial \varepsilon} \int_{ \pm \infty}^{0} e^{-A_{j j^{\sigma}}}\left[B_{j k}(\psi, \varepsilon)+\varepsilon H_{j k}^{\nu}(\psi, \varepsilon)\right] e^{A_{k k} \sigma} d \sigma \\
& =\int_{ \pm \infty}^{0} e^{-A_{j j} \sigma}\left[\left\{\left(\frac{\partial}{\partial \theta_{1}} B_{j k}\right)(\psi, \varepsilon)\right\} \frac{\partial}{\partial \varepsilon} \psi_{1}+\cdots\right. \\
& \left.\quad+\left\{\left(\frac{\partial}{\partial \theta_{m}} B_{j k}\right)(\psi, \varepsilon)\right)\right\} \frac{\partial}{\partial \varepsilon} \psi_{m}+\left\{\left(\frac{\partial}{\partial \varepsilon} B_{j k}\right)(\psi, \varepsilon)\right\}+H_{j k}^{\nu}(\psi, \varepsilon) \\
& \quad+\varepsilon\left\{\left(\frac{\partial}{\partial \theta_{1}} H_{j k}^{\nu}\right)(\psi, \varepsilon)\right\} \frac{\partial}{\partial \varepsilon} \psi_{1}+\cdots+\varepsilon\left\{\left(\frac{\partial}{\partial \theta_{m}} H_{j k}^{\nu}\right)(\psi, \varepsilon)\right\} \frac{\partial}{\partial \varepsilon} \psi_{m} \\
& \left.\quad+\varepsilon\left\{\left(\frac{\partial}{\partial \varepsilon} H_{j k}^{\nu}\right)(\psi, \varepsilon)\right\}\right] e^{A_{k k} \sigma} d \sigma .
\end{aligned}
$$

Define

$$
\begin{aligned}
\|\cdot\|_{0} & =\sup _{(\theta, \varepsilon) \in N_{\delta_{1}}}|\cdot| \\
\|\cdot\|_{1} & =\max _{0 \leqq|\rho| \leqq 1} \sup _{(\theta, \varepsilon) \in N_{\delta_{1}}}\left|D_{(\theta, \varepsilon)}^{\rho}(\cdot)\right| .
\end{aligned}
$$

For the case $j<k$ we have from (14), (16), (17) 


$$
\begin{aligned}
& \left|\frac{\partial}{\partial \theta_{l}} \int_{-\infty}^{0} \cdots d \sigma\right| \leqq K_{2}^{2} \int_{-\infty}^{0} e^{\left(\mu_{k}-\tilde{\mu}_{j}\right) \sigma} m\left\{\|B\|_{1}+|\varepsilon|\left\|H^{\nu}\right\|_{1}\right\} e^{|\varepsilon| K_{1} \mid \sigma} d \sigma \\
& \left|\frac{\partial}{\partial \varepsilon} \int_{-\infty}^{0} \cdots d \sigma\right| \leqq K_{2}^{2} \int_{-\infty}^{0} e^{\left(\mu_{k}-\tilde{\mu}_{j}\right) \sigma}\left[m\left\{\|B\|_{1}+|\varepsilon|\left\|H^{\nu}\right\|_{1}\right\}\right. \\
& \left.\cdot K_{1}|\sigma| e^{|\varepsilon| K_{1}|\sigma|}+\|B\|_{1}+\left\|H^{\nu}\right\|_{0}+|\varepsilon|\left\|H^{\nu}\right\|_{1}\right] d \sigma
\end{aligned}
$$

uniformly in $(\theta, \varepsilon) \in N_{\tilde{o}_{1}}$. Similar inequalities hold for the case $j>k$. If we restrict $\delta_{1}$ so that

$$
\begin{array}{ll}
\mu_{k}-\tilde{\mu}_{j}-\delta_{1} K_{1}>0 & (1 \leqq j<k \leqq n) \\
\tilde{\mu}_{k}-\mu_{j}+\delta_{1} K_{1}<0 & (1 \leqq k<j \leqq n),
\end{array}
$$

then inequality (19) shows that $\partial / \partial \theta_{l}(l=1, \cdots, m), \partial / \partial \varepsilon$ in fact do commute with integration and that (8), (9) determine $Q^{\nu}, H^{\nu}, P^{\nu}(\nu=1$, $2, \cdots)$ iteratively as $C^{1}$ functions of $(\theta, \varepsilon)$ defined on $N_{\delta_{1}}, \delta_{1}$ restricted by (20), with $Q^{\nu}, H^{\nu}, P^{\nu},\left(Q^{\nu}, H^{\nu}, P^{\nu}\right)_{(\theta, \varepsilon)}$ uniformly bounded on $N_{\delta_{1}}$ for each $\nu$ fixed.

We now consider the problem of convergence. With $\delta_{1}$ further restricted, if necessary, we will show that $\left(Q^{\nu}, H^{\nu}, P^{\nu}\right) \rightarrow(Q, H, P)$ in the $C^{1}$ topology on $N_{\hat{o}_{1}}$ as $\nu \rightarrow+\infty$. From (8a)

$$
\begin{array}{ll}
\left\|Q^{\nu+1}-Q^{\nu}\right\|_{0} \leqq \delta_{1} K_{3}\left\|P^{\nu+1}-P^{\nu}\right\|_{0} & (\nu=0,1, \cdots) \\
\left\|Q^{\nu-1}-Q^{\nu}\right\|_{1} \leqq K_{3}\left\|P^{\nu+1}-P^{\nu}\right\|_{1} & (\nu=0,1, \cdots) .
\end{array}
$$

From (8b)

$$
\begin{aligned}
& H^{\nu+1}-H^{\nu}=B\left(P^{\nu}-P^{\nu-1}\right)-P^{\nu}\left(Q^{\nu}-Q^{\nu-1}\right)-\left(P^{\nu}-P^{\nu-1}\right) Q^{\nu-1} \\
&\left\|H^{\nu+1}-H^{\nu}\right\|_{0} \leqq\left\{\|B\|_{0}\right.\left.+\left\|P^{\nu}\right\|_{0}+\left\|Q^{\nu-1}\right\|_{0}\right\}\left\{\left\|P^{\nu}-P^{\nu-1}\right\|_{0}\right. \\
&\left.+\left\|Q^{\nu}-Q^{\nu-1}\right\|_{0}\right\} \\
&\left\|H^{\nu+1}-H^{\nu}\right\|_{1} \leqq 2\left\{\|B\|_{1}\right.\left.+\left\|P^{\nu}\right\|_{1}+\left\|Q^{\nu-1}\right\|_{1}\right\}\left\{\left\|P^{\nu}-P^{\nu-1}\right\|_{1}\right. \\
&\left.+\left\|Q^{\nu}-Q^{\nu-1}\right\|_{1}\right\}_{1} \quad(\nu=1,2, \cdots) .
\end{aligned}
$$

From (9) for the case $j<k$

$$
\begin{aligned}
& \left|P_{j k}^{\nu+1}-P_{j k}^{\nu}\right| \\
& \quad=\left|\int_{-\infty}^{0} e^{-A_{j j} \sigma} \varepsilon\left\{H_{j k}^{\nu+1}(\psi, \varepsilon)-H_{j k}^{\nu}(\psi, \varepsilon)\right\} e^{A_{k k} \sigma} d \sigma\right| \\
& \quad \leqq \int_{-\infty}^{0} K_{2} e^{-\tilde{\mu}_{j} \sigma}|\varepsilon|\left\|H^{\nu+1}-H^{\nu}\right\|_{0} K_{2} e^{\mu_{k} \sigma} d \sigma \\
& \quad \leqq|\varepsilon| K_{4}\left\|H^{\nu+1}-H^{\nu}\right\|_{0} \quad(\nu=1,2, \cdots) .
\end{aligned}
$$

A similar inequality holds for the case $j>k$ so that

$$
\left\|P^{\nu+1}-P^{\nu}\right\|_{0} \leqq \delta_{1} K_{5}\left\|H^{\nu+1}-H^{\nu}\right\|_{0} \quad(\nu=1,2, \cdots) .
$$

Combining (21), (22), (23) we obtain 


$$
\begin{aligned}
& \left\|P^{\nu+1}-P^{\nu}\right\|_{0} \\
& \quad \leqq \delta_{1} K_{5}\left\{\|B\|_{0}+\left\|P^{\nu}\right\|_{0}+\left\|Q^{\nu-1}\right\|_{0}\right\}\left\{1+\delta_{1} K_{3}\right\}\left\|P^{\nu}-P^{\nu-1}\right\|_{0} \\
& \quad(\nu=1,2, \cdots) .
\end{aligned}
$$

From (8), (9) we observe that $Q^{0}, P^{1}$ are defined on $N_{\delta}$ and that on $N_{\delta}$ the inequality

$$
\left|Q^{0}(\theta, \varepsilon)\right| \leqq|B(\theta, \varepsilon)|
$$

holds. Define

$$
K_{6}=2 \sup _{(\theta, \varepsilon) \in N_{\delta}}|B(\theta, \varepsilon)|+3 \sup _{(\theta, \varepsilon) \in N_{\delta}}\left|P^{1}(\theta, \varepsilon)\right| .
$$

Define $V_{\delta_{1}}$ as a subset of the positive integers,

$$
V_{\delta_{1}}=\left\{\nu \mid\|B\|_{0}+\left\|P^{\nu}\right\|_{0}+\left\|Q^{\nu-1}\right\|_{0} \leqq K_{6}\right\} \text {. }
$$

From (24), (26), (27)

$$
\left\|P^{\nu+1}-P^{\nu}\right\|_{0} \leqq \delta_{1} K_{5} K_{6}\left\{1+\delta_{1} K_{3}\right\}\left\|P^{\nu}-P^{\nu-1}\right\|_{0}, \quad \nu \varepsilon V_{\delta_{1}} .
$$

Now restrict $\delta_{1}$ further, if necessary, so that

$$
\begin{gathered}
\delta_{1} K_{3}<\frac{1}{2} \\
\delta_{1} K_{5} K_{6}\left\{1+\delta_{1} K_{3}\right\}<\frac{1}{2} ;
\end{gathered}
$$

then from (21), (25), (26), (28), (29) it follows that

$$
\begin{array}{ll}
\left\|P^{\nu}\right\|_{0} \leqq 2\left\|P^{1}\right\|_{0} & (\nu=1,2, \cdots) \\
\left\|Q^{\nu-1}\right\|_{0} \leqq\|B\|_{0}+\left\|P^{1}\right\|_{0} & (\nu=1,2, \cdots) \\
\|B\|_{0}+\left\|P^{\nu}\right\|_{0}+\left\|Q^{\nu-1}\right\|_{0} \leqq K_{6} & (\nu=1,2, \cdots)
\end{array}
$$

so that by (27)

$$
V_{\delta_{1}}=\{\text { all positive integers }\}
$$

and

$$
\left(Q^{\nu}, H^{\nu}, P^{\nu}\right) \longrightarrow(Q, H, P)
$$

in the $C^{0}$ topology on $N_{\delta_{1}}$ as $\nu \rightarrow+\infty$.

In order to show that our sequence converges in the $C^{1}$ topology we will need $C^{0}$ estimates on the rate of convergence of $\left(H^{\nu+1}-H^{\nu}\right)$, and we will restrict $\delta_{1}$ further, if necessary. From (9), (14) 


$$
\begin{aligned}
& \frac{\partial}{\partial \theta_{l}} P_{j k}^{\nu+1}-\frac{\partial}{\partial \theta_{l}} P_{j k}^{\nu} \\
&= \int_{ \pm \infty}^{0} e^{-A_{j j} \sigma}\left[\varepsilon\left\{\left(\frac{\partial}{\partial \theta_{1}} H_{j k}^{\nu+1}\right)(\psi, \varepsilon)-\left(\frac{\partial}{\partial \theta_{1}} H_{j k}^{\nu}\right)(\psi, \varepsilon)\right\} \frac{\partial}{\partial \theta_{l}} \psi_{1}\right. \\
&\left.+\cdots+\varepsilon\left\{\left(\frac{\partial}{\partial \theta_{m}} H_{j k}^{\nu+1}\right)(\psi, \varepsilon)-\left(\frac{\partial}{\partial \theta_{m}} H_{j k}^{\nu}\right)(\psi, \varepsilon)\right\} \frac{\partial}{\partial \theta_{l}} \psi_{m}\right] e^{A_{k k} \sigma} d \sigma \\
& \frac{\partial}{\partial \varepsilon} P_{j k}^{\nu+1}-\frac{\partial}{\partial \varepsilon} P_{j k}^{\nu} \\
&= \int_{ \pm \infty}^{0} e^{-A_{j j} \sigma}\left[\left\{H_{j k}^{\nu+1}(\psi, \varepsilon)-H_{j k}^{\nu}(\psi, \varepsilon)\right.\right. \\
&+\varepsilon\left\{\left(\frac{\partial}{\partial \theta_{1}} H_{j k}^{\nu+1}\right)(\psi, \varepsilon)-\left(\frac{\partial}{\partial \theta_{1}} H_{j k}^{\nu}\right)(\psi, \varepsilon)\right\} \frac{\partial}{\partial \varepsilon} \psi_{1} \\
&+\cdots+\varepsilon\left\{\left(\frac{\partial}{\partial \theta_{m}} H_{j k}^{\nu+1}\right)(\psi, \varepsilon)-\left(\frac{\partial}{\partial \theta_{m}} H_{j k}^{\nu}\right)(\psi, \varepsilon)\right\} \frac{\partial}{\partial \varepsilon} \psi_{m} \\
&\left.+\varepsilon\left\{\left(\frac{\partial}{\partial \varepsilon} H_{j k}^{\nu+1}\right)(\psi, \varepsilon)-\left(\frac{\partial}{\partial \varepsilon} H_{j k}^{\nu}\right)(\psi, \varepsilon)\right\}\right] e^{A}{ }^{A} k k^{\sigma} d \sigma \\
& \| P^{\nu+1}- P^{\nu}\left\|_{1} \leqq \delta_{1} K_{7}\right\| H^{\nu+1}-H^{\nu}\left\|_{1}+K_{7}\right\| H^{\nu+1}-H^{\nu} \|_{0} \\
&(\nu 1) \quad(\nu=1,2, \cdots) .
\end{aligned}
$$

Combining (21), (22), (31)

$$
\begin{aligned}
\left\|P^{\nu+1}-P^{\nu}\right\|_{1} \leqq \delta_{1} K_{7} 2\left\{\|B\|_{1}+\left\|P^{\nu}\right\|_{1}+\left\|Q^{\nu-1}\right\|_{1}\right\} & \\
\cdot\left\{1+K_{3}\right\}\left\|P^{\nu}-P^{\nu-1}\right\|_{1}+K_{7} \| H^{\nu+1}- & H^{\nu} \|_{0} \\
& (\nu=1,2, \cdots) .
\end{aligned}
$$

From (21), (22), (26), (27), (28), (30)

$$
\left\|H^{\nu+1}-H^{\nu}\right\|_{0} \leqq K_{8}\left\|P^{\nu}-P^{\nu-1}\right\|_{0} \leqq 2^{-\nu+1} K_{8}\left\|P^{1}\right\|_{0}
$$

$$
(\nu=1,2, \cdots) \text {, }
$$

and combining (32), (33)

$$
\begin{aligned}
\left\|P^{\nu+1}-P^{\nu}\right\|_{1} \leqq \delta_{1} K_{7} 2\left\{\|B\|_{1}+\left\|P^{\nu}\right\|_{1}+\left\|Q^{\nu-1}\right\|_{1}\right\} \\
\cdot\left\{1+K_{3}\right\}\left\|P^{\nu}-P^{\nu-1}\right\|_{1}+2^{-\nu+1} K_{7} K_{8}\left\|P^{1}\right\|_{0} \\
\quad(\nu=1,2, \cdots) .
\end{aligned}
$$

Define

$$
\begin{aligned}
K_{9} & =2\|B\|_{1}+2\left(K_{3}+1\right)\left\|P^{1}\right\|_{1}+\left(2 K_{3}+3\right) K_{10} \\
K_{10} & =2 K_{7} K_{8}\left\|P^{1}\right\|_{0} .
\end{aligned}
$$

Although below it may be necessary to restrict $\delta_{1}$ further, $K_{9}$ and $K_{10}$ are computed using $\delta_{1}$ which satisfies (29) and therefore $K_{9}$ and $K_{10}$ are fixed constants for the remainder of the proof. 
Define $V_{\delta_{1}}^{1}$ as a subset of the positive integers

$$
V_{\delta_{1}}^{1}=\left\{\nu \mid\|B\|_{1}+\left\|P^{\nu}\right\|_{1}+\left\|Q^{\nu-1}\right\|_{1} \leqq K_{9}\right\} \text {. }
$$

Now restrict $\delta_{1}$ further, if necessary, so that

$$
2 \delta_{1} K_{7} K_{9}\left\{1+K_{3}\right\}<\frac{1}{4} .
$$

From (34), (35), (36), (37)

$$
\left\|P^{\nu+1}-P^{\nu}\right\|_{1} \leqq \frac{1}{4}\left\|P^{\nu}-P^{\nu-1}\right\|_{1}+\left(\frac{1}{2}\right)^{\nu} K_{16}, \quad \nu \in V_{\delta_{1}}^{1} .
$$

From (38) by induction (as long as $1,2, \cdots, \nu \in V_{\delta 1}^{1}$ )

$$
\begin{gathered}
\left\|P^{\nu+1}-P^{\nu}\right\|_{1} \leqq\left(\frac{1}{4}\right)^{\nu}\left\|P^{1}\right\|_{1}+\left\{\left(\frac{1}{2}\right)^{2 \nu-1}+\left(\frac{1}{2}\right)^{2 \nu-2}\right. \\
\left.+\cdots+\left(\frac{1}{2}\right)^{\nu}\right\} K_{10} \leqq\left(\frac{1}{4}\right)^{\nu}\left\|P^{1}\right\|_{1}+\left(\frac{1}{2}\right)^{\nu-1} K_{10} \\
\left\|P^{\nu}\right\|_{1} \leqq\left\{\left(\frac{1}{4}\right)^{\nu-1}+\left(\frac{1}{4}\right)^{\nu-2}+\cdots+\frac{1}{4}+1\right\}\|P\|_{1} \\
+\left\{\left(\frac{1}{2}\right)^{\nu-2}+\left(\frac{1}{2}\right)^{\nu-3}+\cdots+\frac{1}{2}+1\right\} K_{10}+K_{10}
\end{gathered}
$$

and from (21), (25), (39)

$$
\begin{aligned}
\left\|Q^{\nu-1}\right\|_{1} & \leqq\left\|Q^{\nu-1}-Q^{\nu-2}\right\|_{1}+\cdots+\left\|Q^{1}-Q^{0}\right\|_{1}+\left\|Q^{3}\right\|_{1} \\
& \leqq K_{3}\left[\left\|P^{\nu-1}-P^{\nu-2}\right\|_{1}+\cdots+\left\|P^{1}-P^{0}\right\|_{1}\right]+\|B\|_{1} \\
& \leqq K_{3}\left[\left\{\left(\frac{1}{4}\right)^{\nu-2}+\cdots+\frac{1}{4}+1\right\}\left\|P^{1}\right\|_{1}\right. \\
& \left.+\left\{\left(\frac{1}{2}\right)^{\nu-3}+\cdots+\frac{1}{2}+1\right\} K_{10}\right]+\|B\|_{1} .
\end{aligned}
$$

Hence from (35), (36), (40), (41)

$$
V_{\delta_{1}}^{1}=\{\text { all positive integers }\}
$$

and from (21), (22), (39)

$$
\left(Q^{\nu}, H^{\nu}, P^{\nu}\right) \longrightarrow(Q, H, P)
$$

in the $C^{1}$ topology on $N_{\delta_{1}}$ as $\nu \rightarrow+\infty$. The assertation concerning multiple periodicity follows from standard arguments. This completes the proof of Theorem 1.

Using the proof method developed for Theorem 1, we can prove

Theorem 2. For system (1) where $b(\theta, \varepsilon)$ and $B(\theta, \varepsilon)$ are 
$C^{k}(1 \leqq k<\infty)$ with uniformly bounded derivatives on some $N_{\delta}$, the change of variables (3) and the transformed system (4) are also $C^{k}$ with uniformly bounded derivatives on some $N_{\delta_{1}}$ provided $0<\delta_{1} \leqq \delta$ is sufficiently small.

4. The analytic case. In this section we first construct a formal power series change of variables and then give a counterexample to show that in general the power series does not converge. The procedure is then modified in Theorem 3.

Consider the real analytic system of ordinary differential equations

$$
\begin{aligned}
& \dot{\theta}=a+\varepsilon b(\theta, \varepsilon) \\
& \dot{x}=(A+\varepsilon B(\theta, \varepsilon)) x
\end{aligned}
$$

which is the same as (1) except that $b, B$ are defined and analytic on

$$
N_{\grave{o}}^{0}=\{(\theta, \varepsilon)|| \mathscr{J}(\theta)|<\delta,| \varepsilon \mid<\delta\}
$$

and $b_{(\theta, \varepsilon)}, B_{(\theta, \varepsilon)}$ are uniformly bounded on $N_{\dot{o}}^{\circ}$, where $\mathscr{F} \equiv$ imaginary part, $\mathscr{I}(\theta) \equiv\left(\mathscr{J}\left(\theta_{1}\right), \cdots, \mathscr{J}\left(\theta_{m}\right)\right)$. If we look for an analytic change of variables

$$
x=(I+\varepsilon P(\theta, \varepsilon)) y
$$

such that

$$
\dot{y}=(A+\varepsilon Q(\theta, \varepsilon)) y,
$$

where $P$ and $Q$ are defined and analytic on some region $N_{\dot{\delta}_{1}}^{\circ}, P$ and $Q$ satisfying (5); then we must solve $(7 \mathrm{a}, \mathrm{b})$. Because (42) is analytic, it is natural to write (see the left side of $7 \mathrm{~b}$ )

$$
\dot{P}_{j k}=\sum_{l=1}^{m}\left\{a_{l}+\varepsilon b_{l}(\theta, \varepsilon)\right\} \frac{\partial}{\partial \theta_{l}} P_{j k}
$$

where $\theta=\left(\theta_{1}, \cdots, \theta_{m}\right), a=\left(a_{1}, \cdots, a_{m}\right), b=\left(b_{1}, \cdots, b_{m}\right)$. Then, rather than $(7 \mathrm{a}, \mathrm{b})$ we try to solve

$$
\begin{aligned}
Q_{j j}= & B_{j j}+\varepsilon \sum_{l=1}^{n} B_{j l} P_{l j} \quad(j=1, \cdots, n) \\
\sum_{l=1}^{m} a_{l} \frac{\partial}{\partial \theta_{l}} P_{j k}= & A_{j j} P_{j k}-P_{j k} A_{k k}+B_{j k} \\
& +\varepsilon \sum_{l=1}^{m} B_{j l} P_{l k}-\varepsilon P_{j k} Q_{k k}-\varepsilon \sum_{l=1}^{m} b_{l} \frac{\partial}{\partial \theta_{l}} P_{j k} \\
& \quad(j, k=1, \cdots, n ; j \neq k) .
\end{aligned}
$$

Since $B$ is analytic, it has a power series expansion

$$
B(\theta, \varepsilon)=\sum_{g=0}^{\infty} \varepsilon^{g} B^{g}(\theta) \text {. }
$$


(The super-scripts on $B$ designate matrix coefficients in the expansion, not powers of the matrix $B$; this remark will hold below relative to $P, Q$, etc.) If we assume $P$ and $Q$ have power series expansions

$$
\begin{aligned}
& P(\theta, \varepsilon)=\sum_{g=0}^{\infty} \varepsilon^{g} P^{g}(\theta) \\
& Q(\theta, \varepsilon)=\sum_{g=0}^{\infty} \varepsilon^{g} Q^{g}(\theta),
\end{aligned}
$$

then in (43) we can solve for the coefficients of $P$ and $Q$ recursively. Equating corresponding powers of $\varepsilon$ in $(43 b)$ we have

$$
\sum_{l=1}^{m} a_{l} \frac{\partial}{\partial \theta_{l}} P_{j k}^{g}=A_{j j} P_{j k}^{g}-P_{j k}^{g} A_{k k}+Z_{j k}^{g}
$$

where $Z_{j}^{g}=Z_{j k}^{g}(\theta)$ is some known matrix function of $\theta ; Z^{g}$ depends only on the coefficients of $P$ and $Q$ of degree less than $g$. The unique bounded solution of (44) in given by

$$
P_{J k}^{g}(\theta)=\int_{ \pm \infty}^{0} e^{-1_{j} j^{\sigma}} Z_{j k}^{g}(\theta+a \sigma) e^{A k k^{\sigma}} d \sigma
$$

where the lower limit of integration is chosen $+\infty$ for $A_{k k}<A_{j j}$ and $-\infty$ for $A_{j j}<A_{k k}$ with $<$ the order relation in (2).

Thus from (43a) and (45) we can construct the formal power series for $P$ and $Q$ recursively. If $\operatorname{dim} \theta>1$, the following counter example shows that in general the formal power series for $P$ and $Q$ need not converge.

Consider the real analytic, four dimensional system of differential equations

$$
\begin{aligned}
& \dot{\theta}=a+\varepsilon b \\
& \dot{x}=(A+\varepsilon B(\theta)) x
\end{aligned}
$$

where $\theta=\left(\theta_{1}, \theta_{2}\right), a=\left(a_{1}, a_{2}\right), b=(1,0), x=\left(x_{1}, x_{2}\right)$

$$
A=\left(\begin{array}{cc}
\lambda_{1} & 0 \\
0 & \lambda_{2}
\end{array}\right), \quad B=\left(\begin{array}{cc}
0 & B_{12}(\theta) \\
0 & 0
\end{array}\right),
$$

with $\lambda_{1} \neq \lambda_{2}$. From $(43 a, b)$

$$
\begin{aligned}
Q_{11}= & B_{11}+\varepsilon\left\{B_{11} P_{11}+B_{12} P_{21}\right\}=\varepsilon P_{21} \\
Q_{22}= & B_{22}+\varepsilon\left\{B_{21} P_{12}+B_{22} P_{22}\right\}=0 \\
\sum_{l=1}^{2} a_{l} \frac{\partial}{\partial \theta_{l}} P_{12}= & \left(\lambda_{1}-\lambda_{2}\right) P_{12}+B_{12} \\
& +\varepsilon\left\{B_{11} P_{12}+B_{12} P_{22}\right\}-\varepsilon P_{12} Q_{22}-\varepsilon \frac{\partial}{\partial \theta_{1}} P_{12} \\
= & \left(\lambda_{1}-\lambda_{2}\right) P_{12}+B_{12}-\varepsilon \frac{\partial}{\partial \theta_{1}} P_{12}
\end{aligned}
$$




$$
\begin{aligned}
\sum_{l=1}^{2} a_{l} \frac{\partial}{\partial \theta_{l}} P_{21}= & \left(\lambda_{2}-\lambda_{1}\right) P_{21}+B_{21} \\
& +\varepsilon\left\{B_{21} P_{11}+B_{22} P_{21}\right\}-\varepsilon P_{21} Q_{11}-\varepsilon \frac{\partial}{\partial \theta_{1}} P_{21} \\
= & \left(\lambda_{2}-\lambda_{1}\right) P_{21}-\left(\varepsilon P_{21}\right)^{2}-\varepsilon \frac{\partial}{\partial \theta_{1}} P_{21}
\end{aligned}
$$

From (47d), (45) we have $P_{21} \equiv 0$; from $(47 \mathrm{a}, \mathrm{b}) Q \equiv 0$. Thus we have only $P_{12}$ to compute in $(47 \mathrm{c})$.

Suppose $B_{12}$ has the Fourier series representation

$$
B_{12}(\theta)=\sum_{\nu_{1}, \nu_{2}=-\infty}^{+\infty} \beta_{\nu_{1}, \nu_{2}} e^{i\left[\nu_{1} \theta_{1}+\nu_{2} \theta_{2}\right\}} .
$$

If $\psi=\left(\psi_{1}, \psi_{2}\right), \psi=\psi(t, \theta, \varepsilon)$ is the unique solution of the $\theta$-equation in (46) with initial condition $\theta$ at $t=0$, then

$$
\begin{aligned}
& \psi_{1}(t, \theta, \varepsilon)=\theta_{1}+\left(a_{1}+\varepsilon\right) t \\
& \psi_{2}(t, \theta, \varepsilon)=\theta_{2}+a_{2} t .
\end{aligned}
$$

From (47c)

$$
\begin{gathered}
\frac{d}{d t} P_{12}(\psi(t, \theta, \varepsilon))=\left(\lambda_{1}-\lambda_{2}\right) P_{12}(\psi(t, \theta, \varepsilon)) \\
+\sum \beta_{\nu_{1}, \nu_{2}} e^{i\left(\nu_{1}\left(\theta_{1}+\left\langle a_{1}+\varepsilon\right) t\right)+\nu_{2}\left(\theta_{2}+a_{2} t\right)\right\}}
\end{gathered}
$$

and therefore, when $\varepsilon$ is real,

$$
P_{12}(\theta, \varepsilon)=\sum\left[\lambda_{2}-\lambda_{1}+i\left\{\nu_{1}\left(a_{1}+\varepsilon\right)+\nu_{2} a_{2}\right\}\right]^{-1} \beta_{\nu_{1}, \nu_{2}} e^{i\left\langle\nu_{1} \theta_{1}+\nu_{2} \theta_{2}\right\}} .
$$

But since

$$
\left[\lambda_{2}-\lambda_{1}+i\left\{\nu_{1}\left(a_{1}+\varepsilon\right)+\nu_{2} a_{2}\right\}\right]=0
$$

when

$$
\varepsilon=\nu_{1}^{-1}\left[-i\left(\lambda_{2}-\lambda_{1}\right)-\nu_{2} \alpha_{2}\right]-\alpha_{1},
$$

we conclude that in general $P_{12}(\theta, \varepsilon)$ can not be analytic in $\varepsilon$.

Rather than (42) we consider the real system of ordinary differential equations

$$
\begin{aligned}
& \dot{\theta}=a+\tilde{\varepsilon} b(\theta, \tilde{\varepsilon}) \\
& \dot{x}=(A+\varepsilon B(\theta, \varepsilon)) x
\end{aligned}
$$

where $\theta, a, b$ are real vectors; $a$ is a constant vector; $\tilde{\varepsilon}$ is a real perturbation parameter; $b$ is defined and $C^{k}(1 \leqq k<\infty)$ on

$$
N_{\delta}^{1}=\left\{(\theta, \tilde{\varepsilon}) \mid \theta \in R^{m},-\delta<\tilde{\varepsilon}<\delta\right\}
$$


with $m=\operatorname{dim} \theta, R^{m} \equiv m$-dimensional euclidean space; $b$ and all its derivatives of order less than or equal to $k$ with respect to the components of $(\theta, \tilde{\varepsilon})$ are uniformly bounded on $N_{\delta}^{1} ; A$ is the real, constant matrix given in (1); $\varepsilon$ is a complex perturbation parameter; $B$ is real analytic in $\varepsilon$ and $C^{k}$ in $(\theta, \varepsilon)$ on

$$
N_{\tilde{o}}^{2}=\left\{(\theta, \varepsilon)\left|\theta \in R^{m},\right| \varepsilon \mid<\delta\right\}
$$

and has a power series expansion on $N_{\hat{o}}^{2}$,

$$
B(\theta, \varepsilon)=\sum_{g=0}^{\infty} \varepsilon^{g} B^{g}(\theta) ;
$$

$B$ and all its derivatives of order less than or equal to $k$ with respect to the components of $\theta$ are uniformly bounded on $N_{\tilde{\delta}}^{2}$, and these derivatives of $B$ are equal to the term by term derivatives of the power series for $B$.

THEOREM 3. For system (48) there exists a unique change of variables

$$
x=(I+\varepsilon P(\theta, \tilde{\varepsilon}, \varepsilon)) y
$$

such that

$$
\dot{y}=(A+\varepsilon Q(\theta, \tilde{\varepsilon}, \varepsilon)) y
$$

where $I$ is the identity matrix; $P$ and $Q$ are real analytic in $\varepsilon$ and $C^{k}$ in $(\theta, \tilde{\varepsilon}, \varepsilon)$ on some

$$
N_{\delta_{1}}^{3}=\left\{(\theta, \tilde{\varepsilon}, \varepsilon)\left|\theta \in R^{m},-\delta_{1}<\tilde{\varepsilon}<\delta_{1},\right| \varepsilon \mid<\delta_{1}\right\}
$$

with $0<\delta_{1} \leqq \delta$ sufficiently small; $P$ and $Q$ have power series expansions on $N_{\tilde{o}_{1}}^{3}$,

$$
\begin{aligned}
& P(\theta, \tilde{\varepsilon}, \varepsilon)=\sum_{g=0}^{\infty} \varepsilon^{g} P^{g}(\theta, \tilde{\varepsilon}) \\
& Q(\theta, \tilde{\varepsilon}, \varepsilon)=\sum_{g=0}^{\infty} \varepsilon^{g} Q^{g}(\theta, \tilde{\varepsilon}) ;
\end{aligned}
$$

$P$ and $Q$ and all their derivatives of order less than or equal to $k$ with respect to the components of $(\theta, \tilde{\varepsilon})$ are uniformly bounded on $N_{\tilde{o}_{1}}^{3}$, and these derivatives of $P$ and $Q$ are equal to the term by term derivatives of the power series for $P$ and $Q$, respectfully; with $P$ and $Q$ partitioned into sub-matrices similar to $A$,

$$
\begin{array}{rlrl}
P_{j j}(\theta, \tilde{\varepsilon}, \varepsilon) & \equiv 0 & (j=1, \cdots, n) \\
Q_{j k}(\theta, \tilde{\varepsilon}, \varepsilon) \equiv 0 & (j, k=1, \cdots, n ; j \neq k)
\end{array}
$$

if system (48) has multiple period $\omega$ in $\theta$, then $P$ and $Q$ also have multiple period $\omega$ in $\theta$. 
Proof. Following the proof method of Theorem 1, we obtain from (49), (50), (52)

$$
\begin{aligned}
& Q_{j j}=B_{j j}+\varepsilon \sum_{l=1}^{n} B_{j l} P_{l j} \quad(j=1, \cdots, n) \\
& \dot{P}_{j k}=A_{j j} P_{j k}-P_{j k} A_{k k}+B_{j k} \\
& +\varepsilon \sum_{l=1}^{n} B_{j l} P_{l k}-\varepsilon P_{j k} Q_{k k} \quad(j, k=1, \cdots, n ; j \neq k) .
\end{aligned}
$$

Assuming $P$ and $Q$ have power series representations (51) with (as of yet) undetermined coefficients, we obtain upon equating coefficients of $\varepsilon^{g}$ in $(53)$

$$
\begin{aligned}
Q_{j j}^{g} & =B_{j j}^{g}+W_{j j}^{g} \\
\dot{P}_{j k}^{g} & =A_{j j} P_{j k}^{g}-P_{j k}^{g} A_{k k}+B_{j k}^{g}+Z_{j k}^{g}
\end{aligned}
$$

where $W^{g}=W^{g}(\theta, \tilde{\varepsilon})$ and $Z^{g}=Z^{g}(\theta, \tilde{\varepsilon})$ are matrices which depend on the coefficients of $P$ and $Q$ of degree less then $g$. To solve (54b) define

$$
\begin{aligned}
P_{j k}^{g}(\theta, \tilde{\varepsilon})= & \int_{ \pm \infty}^{0} e^{-A_{j j^{\sigma}}}\left\{B_{j k}^{g}(\psi(\sigma, \theta, \tilde{\varepsilon}))\right. \\
& \left.+Z_{j k}^{q}(\psi(\sigma, \theta, \tilde{\varepsilon}), \tilde{\varepsilon})\right\} e^{A_{k k} \sigma} d \sigma
\end{aligned}
$$

where $\psi=\psi(t, \theta, \tilde{\varepsilon})$ is the unique solution to the $\theta$-equation in (48) with initial condition $\theta$ at $t=0$. Using (54), (55) we compute the coefficients of $P$ and $Q$ recursively. By restricting $\delta_{1}$ sufficiently, one easily shows that $P^{g}, Q^{g}(g=0,1,2, \cdots)$ have, with respect to the components of $(\theta, \tilde{\varepsilon})$, uniformly bounded derivatives of order less than or equal to $k$. To show that the power series for $P$ and $Q$ converge and that the term-by-term derivatives up to order $k$ of the power series also converge, one uses the proof method of Theorem 1. The assertion concerning multiple periodicity follows from a standard argument. This completes the proof of Theorem 3 .

\section{BIBLIOGRAPHY}

1. E. Coddington and N. Levinson, Theory of Ordinary Differential Equations, McGraw-Hill, 1955.

2. J. Hale, Oscillations in Nonlinear Systems, McGraw-Hill, New York, 1963.

3. A. Kelley, The stable, center-stable, center, center-unstable, unstable manifolds, J. Differential Equations, 3 (1967), 546-570.

4. - Changes of variables near a periodic surface or invariant manifold, Trans. Amer. Math. Soc. (to appear).

5. Y. Sibuya, Nonlinear ordinary differential equations with period coefficients, Funkcialaj Ekvacioj, 1 (1958), 201-204.

6. — On perturbations of periodic solutions, J. Math. Mech. 9 (1960), 771-782. 
7. R. Venti, Linear normal forms of differential equations, J. Differential Equations, 2 (1966), 182-194.

Received April 14, 1967.

UNIVERSITY OF CALIFORNIA

Santa CRUz 


\section{PACIFIC JOURNAL OF MATHEMATICS}

\section{EDITORS}

\section{H. ROYDEN}

Stanford University

Stanford, California
J. DugundJI

Department of Mathematics

Rice University

Houston, Texas 77001

RICHARD ARENS

University of California

Los Angeles, California 90024

Seattle, Washington 98105

\section{ASSOCIATE EDITORS}
E. F. BeCKENBACH
B. H. NeumanN
F. WOLF
K. YOSIDA

\section{SUPPORTING INSTITUTIONS}

\author{
UNIVERSITY OF BRITISH COLUMBIA \\ CALIFORNIA INSTITUTE OF TECHNOLOGY \\ UNIVERSITY OF CALIFORNIA \\ MONTANA STATE UNIVERSITY \\ UNIVERSITY OF NEVADA \\ NEW MEXICO STATE UNIVERSITY \\ OREGON STATE UNIVERSITY \\ UNIVERSITY OF OREGON \\ OSAKA UNIVERSITY \\ UNIVERSITY OF SOUTHERN CALIFORNIA
}

\author{
STANFORD UNIVERSITY \\ UNIVERSITY OF TOKYO \\ UNIVERSITY OF UTAH \\ WASHINGTON STATE UNIVERSITY \\ UNIVERSITY OF WASHINGTON \\ * * * * \\ AMERICAN MATHEMATICAL SOCIETY \\ CHEVRON RESEARCH CORPORATION \\ TRW SYSTEMS \\ NAVAL WEAPONS CENTER
}

Mathematical papers intended for publication in the Pacific Journal of Mathematics should be in typed form or offset-reproduced, double spaced with large margins. Underline Greek letters in red, German in green, and script in blue. The first paragraph or two must be capable of being used separately as a synopsis of the entire paper. It should not contain references to the bibliography. Manuscripts, in duplicate if possible, may be sent to any one of the four editors. All other communications to the editors should be addressed to the managing editor, Richard Arens, University of California, Los Angeles, California 90024.

Each author of each article receives 50 reprints free of charge; additional copies may be obtained at cost in multiples of 50 .

The Pacific Journal of Mathematics is published monthly. Effective with Volume 16 the price per volume (3 numbers) is $\$ 8.00$; single issues, $\$ 3.00$. Special price for current issues to individual faculty members of supporting institutions and to individual members of the American Mathematical Society: $\$ 4.00$ per volume; single issues $\$ 1.50$. Back numbers are available.

Subscriptions, orders for back numbers, and changes of address should be sent to Pacific Journal of Mathematics, 103 Highland Boulevard, Berkeley 8, California.

Printed at Kokusai Bunken Insatsusha (International Academic Printing Co., Ltd.), 7-17, Fujimi 2-chome, Chiyoda-ku, Tokyo, Japan.

PUBLISHED BY PACIFIC JOURNAL OF MATHEMATICS, A NON-PROFIT CORPORATION

The Supporting Institutions listed above contribute to the cost of publication of this Journal, but they are not owners of publishers and have no responsibility for its content or policies. 


\section{Pacific Journal of Mathematics \\ Vol. 25, No. $2 \quad$ October, 1968}

Martin Aigner, On the tetrahedral graph ..................... 219

Gregory Frank Bachelis, Homomorphisms of annihilator Banach

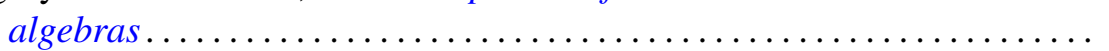

Phillip Alan Griffith, Transitive and fully transitive primary abelian groups.......................................... 249

Benjamin Rigler Halpern, Fixed points for iterates . . . . . . . . . . . . . 255

James Edgar Keesling, Mappings and dimension in general metric spaces ......................................... 277

$\mathrm{Al}$ (Allen Frederick) Kelley, Jr., Invariance for linear systems of ordinary differential equations ................................ 289

Hayri Korezlioglu, Reproducing kernels in separable Hilbert spaces . . . . . 305

Gerson Louis Levin and Wolmer Vasconcelos, Homological dimensions and Macaulay rings ................................. 315

Leo Sario and Mitsuru Nakai, Point norms in the construction of harmonic

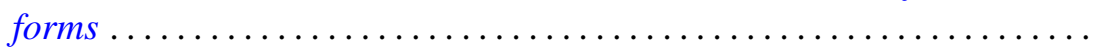

Barbara Osofsky, Noncommutative rings whose cyclic modules have cyclic injective hulls ..................................... 331

Newton Tenney Peck, Extreme points and dimension theory........... 341

Jack Segal, Quasi dimension type. II. Types in 1-dimensional spaces ...... 353

Michael Schilder, Expected values of functionals with respect to the Ito distribution ...

Grigorios Tsagas, A Riemannian space with strictly positive sectional curvature

John Alexander Williamson, Random walks and Riesz kernels . . 